$$
\begin{gathered}
10613 \\
48513 \\
1.32
\end{gathered}
$$

\title{
A High Performance Totally Ordered Multicast Protocol
}

\author{
by Todd Montgomery, Brian Whetten, and Simon Kaplan
}

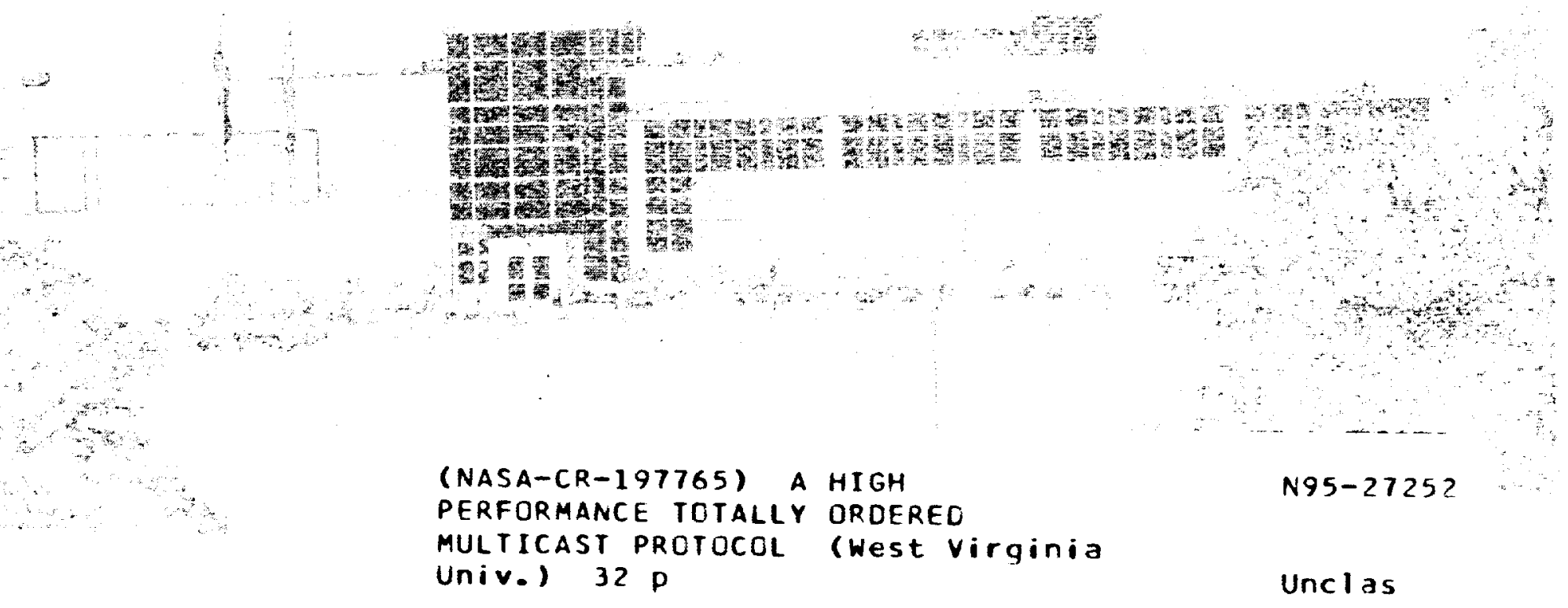

National Aeronautics and Space Administration

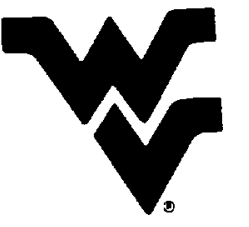

West Virginia University 
According to the terms of Cooperative Agreement $\#$ NCCW-0040, the following approval is granted for distribution of this technical report outside the NASA/WVU Software Research Laboratory

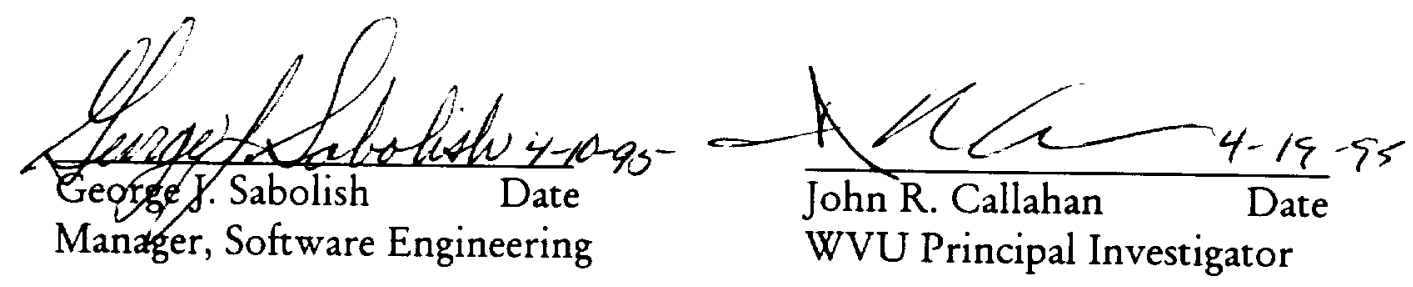




\title{
A HIGH PERFORMANCE TOTALLY ORDERED MULTICAST PROTOCOL
}

\author{
Brian Whetten, University of California at Berkeley (whetten@cs.berkeley.edu) \\ Simon Kaplan, University of Illinois at Champaign-Urbana (kaplan@cs.uiuc.edu) \\ Todd Montgomery, West Virginia University (tmont@cerc.wvu.edu)
}

\begin{abstract}
This paper presents the Reliable Multicast Protocol (RMP). RMP provides a totally ordered, reliable, atomic multicast service on top of an unreliable multicast datagram service such as IP Multicasting. RMP is fully and symmetrically distributed so that no site bears an undue portion of the communication load. RMP provides a wide range of guarantees, from unreliable delivery to totally ordered delivery, to K-resilient, majority resilient, and totally resilient atomic delivery. These QOS guarantees are selectable on a per packet basis. RMP provides many communication options, including virtual synchrony, a publisher/subscriber model of message delivery, a client/server model of delivery, an implicit naming service, mutually exclusive handlers for messages, and mutually exclusive locks.

It has commonly been held that a large performance penalty must be paid in order to implement total ordering--RMP discounts this. On SparcStation10's on a $1250 \mathrm{~KB} / \mathrm{sec}$ Ethernet. RMP provides totally ordered packet delivery to one destination at $842 \mathrm{~KB} / \mathrm{sec}$ throughput and with $3.1 \mathrm{~ms}$ packet latency. The performance stays roughly constant independent of the number of destinations. For two or more destinations on a LAN, RMP provides higher throughput than any protocol that does not use multicast or broadcast.
\end{abstract}

Keywords: Congestion control, internetworking. distributed network algorithms, network reliability

\section{Introduction}

Totally ordered. reliable broadcast and multicast protocols have existed for quite some time [ChMa84], and provide a powerful tool for programming distributed systems and distributed databases [Chang84]. New applications such as Computer Supported Cooperative Work (CSCW) programs, groupware systems and shared tools can also benefit greatly from this service. In the past, these protocols have had problems with performance, efficiency, and/or scalability. It has become a widespread belief that these are inherent problems with a totally ordered reliable multicast protocol [RaLi93]. In part, this concept resulted from the fact that in the past multicasts had to be implemented as a series of unicasts to each 
destination. Recent developments such as the IP Multicasting standard [Deering89] now allow a multicast datagram to be sent to multiple destinations over an internetwork. In the case where all destinations are on the same LAN, one multicast packet to all of them costs the same as a unicast packet to just one.

This paper presents the Reliable Multicast Protocol (RMP) which provides a reliable multicast service on top of unreliable datagram services such as IP and IP Multicasting. RMP is based on a modified version of the token passing protocol proposed by J. M. Chang and N. F. Maxemchuk in [ChMa83] and [ChMa84]. The basic RMP protocol provides what can be thought of as a $\mathrm{N}$-way virtual circuits, called token rings, between groups of processes connected by a multicast medium. It is fully distributed, so that all processes play the same role in communication. While primarily using NACKs for error detection and retransmission. RMP provides true reliability and limits the necessary buffer space by passing a token around the members of a token ring.

RMP provides a wide range of reliability and ordering guarantees on packet delivery, selectable on a per packet basis. In addition to unreliable and reliable but unordered quality of service (QOS) levels. RMP can provide atomic, reliably delivery of packets ordered with respect to each source. It can also efficiently provide delivery of packets in both total and causal order. using causal ordering as defined in [Lamp78]. Totally ordered delivery also provides virtual synchrony, as first defined by the ISIS project [BSS91]. Virtual synchrony guarantees that when new members join or leave a group these operations appear to be atomic, so that the sets of messages delivered before and after each membership change are consistent across all sites. Using K-resilient fault tolerance. RMP can provide total ordering and atomicity guarantees even in the face of site failures and partitions. For a set of packets with a resiliency level of $K$, more than $K$ members of a group have to simultaneously partition away or fail in order to have the possibility of violating the total ordering and atomicity guarantees. By setting $\mathrm{K}$ to a number larger than half the members of a ring and not allowing minority partitions to continue. total ordering, atomicity, and virtual synchrony can be guaranteed in the face of any set of arbitrary partitions and failures.

The basic RMP model of communication is a publisher/subscriber model based on textual token ring names. In the absence of network partitions, any member of a token ring (a subscriber) will receive all packets sent (published) to the token ring associated with that token ring name. RMP also provides a client/server model of communication, where the servers are members of a token ring and the clients are not members, but can communicate with the servers by sending 
multi-RPC packets to the group. These packets may be simply acknowledged after being delivered to the token ring with the requested QoS, or they may be responded to by a single member of the token ring. RMP uses handlers to guarantee that at most one member will respond to a data packet. Each data packet in RMP has an optional handler number associated with it. These correspond to a set of mutually exclusive handler locks which token ring members may hold. The token ring member who holds a given handler lock will be notified upon delivery of a data packet with this handler number that it is supposed to respond to the request. Handler locks are provided in a very efficient way. and can be used for any type of application that requires mutually exclusive locks shared among a group of communicating processes.

A common belief in the research community is that totally ordered reliable multicast protocols are inherently slow. This belief has come about in large part due to the experiences researchers have had with the early versions of ISIS, which for a long time was the only system of this type available. ISIS has since become much faster [BiCl94], but the misconception remains. Experience with RMP belies this concept. RMP was tested on 8 SparcStation10's on a 10 $\mathrm{Mb} / \mathrm{sec}(1250 \mathrm{~KB} / \mathrm{sec})$ Ethernet. In this environment, the throughput to a single destination is $842 \mathrm{~KB} / \mathrm{sec}$, or $67.4 \%$ of the network capacity. For group communication to any group of two or more destinations on a LAN, RMP exceeds not only the maximum throughput of TCP/IP, but any other possible non-multicast and non-broadcast algorithm. This is because both the packet latency and throughput of RMP stay roughly constant as the number of destinations increase. whereas the performance of other algorithms decreases linearly. For a group with 8 destinations. RMP has a $6.8 \mathrm{MB} / \mathrm{sec}$ aggregate throughput, which is 5.4 times the bandwidth of the supporting Ethernet. The throughput for RMP does not significantly change as a factor of the ordering guarantees, but the per packet latency does. A totally ordered packet will on average have a latency approximately twice that of an unordered or source ordered packet, and this increases for $\mathbf{K}$ resilient packets. This QOS for latency tradeoff is fundamental to distributed protocols, which is why RMP allows this tradeoff to be made on a per packet basis. Despite this moderate latency penalty for its total ordering. RMP latency to two or more destinations is still lower than most other protocols. Therefore RMP demonstrates that a fault tolerant, reliable. atomic, fully distributed, totally ordered multicast protocol can actually achieve much better performance in group communication than systems that don't provide these features. 
Section 2 describes the architecture and system usage model of RMP. Section 3 describes the RMP algorithms. Section 4 analyzes the performance of RMP, and section 5 compares RMP to previous work. Finally, section 6 draws our conclusions and outlines future work and section 7 acknowledges all the support we have had for RMP.

\section{System usage and architecture}

RMP is a transport level protocol that provides reliable datagram delivery on top of a unicast or multicast unreliable datagram service. It allows multiple groups of processes to communicate with selectable levels of reliability and ordering through the use of a QOS field in each data packet. RMP supports both implicit and explicit naming as well as publisher/subscriber and client/server models of communication. Finally, RMP allows processes that are not multicast capable to participate in the group communication through the use of forwarding and non-multicast capable flags.

\subsection{RMP entities}

RMP is organized around RMP processes, token rings, and token lists. The basic entity that uses RMP to communicate is called a RMP process. A RMP process typically corresponds to a single UNIX process. application, or "software bus". There may be multiple RMP processes on the same host. Each RMP process is uniquely identified by the IP address of its host concatenated with a UDP port number that may not be reused between processes on a host and is constant over the life of that RMP process. This $\mathrm{D}$, called a RMP process $I D$, is unique across all RMP processes in the internetwork.

A token ring is the basic unit of group communication and message ordering in RMP, and consists of a group of RMP processes that are receiving packets sent to a given IP Multicast address and port. Each RMP process may be a member of multiple token rings, and non-members can reliably send to a token ring and get optional replies from members of a token ring using an RPC like mechanism. Each token ring has a token ring name, which is a text string similar to those used in current Internet host names. Unlike host names, a token ring name identifies a group of members instead of a single host. A token ring identified by a token ring name is not guaranteed to be unique across an entire internetwork, as two groups of RMP processes that join a given IP Multicast address and port with a given token ring name may not overlap due to network partitions or due to non-global multicast time to live levels (TTLs) on packets sent to this address. 
The mapping of token ring names to IP Multicast \{address, port. TTL\} tuples may either be handled by an external multicast address allocation authority such as [PEA94], or it may be handled by RMP. A suggested default mapping policy is to use a hash function to turn the text string into one of a range of multicast addresses (a subset of the class D IP address space, say 24 bits) and a 16 bit port number. The TTL for packets sent to a token ring will be negotiated as part of the membership algorithms for a token ring. Hash collisions to the same port and address are handled by using guaranteed unique token list IDs, as described below.

The membership of a token ring will usually change over time. A given list of the members of a token ring is called a token list. A token list is always created by a single RMP process, and is identified by a token list ID. Similarly to Grapevine[BLNS82], a token list ID consists of an ID that is unique across the breadth of the internetwork concatenated with a counter that is unique across the maximum TTL for that $\mathrm{D}$. The first of a token list ID consists of the RMP process ID of the process that created the token list, and the second half is based on a counter. One counter is maintained for each RMP process. When an RMP process starts, it initializes its counter to the current time in milliseconds, and it increments this counter every time it creates a new token list. By not allowing an RMP process to generate more than one token list per millisecond the process has been in existence and by limiting the lifetime of a token list to $2^{31}$ milliseconds (just under 25 days), we guarantee that a token list $\mathrm{ID}$ is unique as long as the clocks of the generating machines are stable. This guarantee eliminates the need to keep the IP Multicast address and port for a token ring unique across different token rings, although it is desirable to avoid these address collisions. In the case of collisions to the same

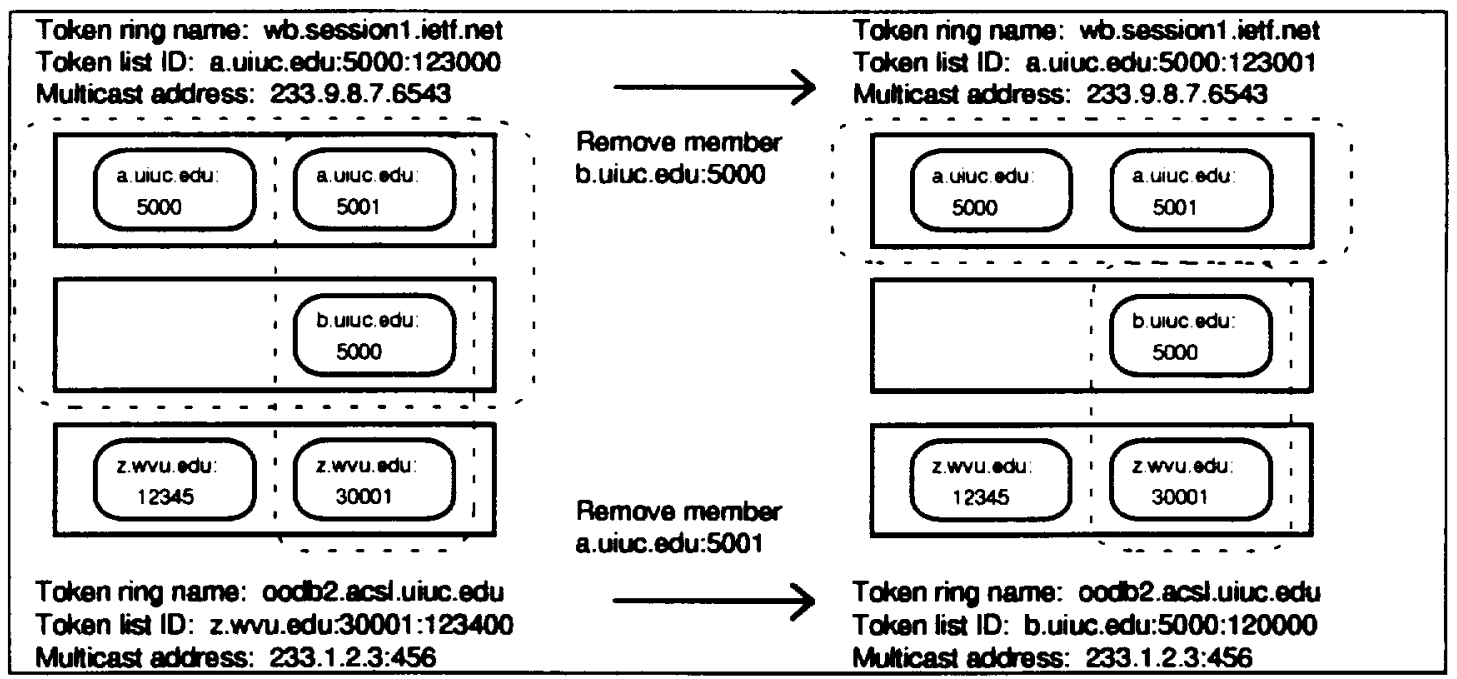

Fgure 2.1: RMP entities 
address and port. RMP will use the token list Ds to filter the packets at each RMP process.

Figure 2.1 shows an example of these entities for a sample scenario with two token rings and five RMP processes spread over three hosts. A new token list $\mathrm{D}$ is generated for both token rings after a member is removed from each. Notice that the new ID does not have to be created by the same RMP process that created the last one for that ring.

\subsection{Atomicity, reliability and ordering guarantees}

Different multicast applications require many different levels of atomicity, reliability, and ordering guarantees. These applications also require different guarantees in the face of site failures or partitions. For example, a CSCW application may need packets to be reliably delivered in order from each source but without total ordering. and will continue even if some sites fail or partition away. On the other hand, a distributed database may require that all packets be delivered in the same total order at all sites, even if some of them partition away. RMP supports a wide range of guarantees on packets by allowing different QoS levels to be specified for packets being sent to a token ring and by allowing applications to specify the minimum size of a partition that can continue to function in the face of failures. The selectable QoS levels are described in figure 2.2. All of the QoS levels build upon previous levels, providing any guarantees that a smaller level provides.

The basic selectable RMP QOS levels are unreliable, reliable, source ordered, and total ordered. They are provided by differing the time at when packets are delivered and enabling or disabling the duplicate detection. NACK, and ACK policies. While throughput should remain similar for the different QoS levels, higher QaS levels increase the latency of packet delivery. For example, in the common case of few dropped packets, source ordered packets have about the same latency as unordered packets, and totally ordered packets have about twice the latency of either.

The unreliable QOS is most similar to UDP traffic. An unreliable packet will be delivered 0,1 , or more times to a destination and there are no ordering guarantees on delivery. A reliably delivered packet will be delivered 1 or more times to each destination. This is particularly useful for providing unordered client-server RPC semantics, as explained in a later section. The source ordered QOS provides the equivalent guarantees of running a TCP socket from each source to each destination. Packets arrive exactly once at each destination in the same order as they were sent from the sender. Total ordered delivery serializes all of the packets to a token ring, delivering all of the packets in the same order at all 
members of the ring. This $Q D S$ is equivalent to running a TCP socket from each source into a central bus which serializes the packets and then sends them out through a separate TCP socket to each destination. Totally ordered packets are also causally ordered, as per Lamport's definition[Lamp78]. All of the QOS levels of source ordered and higher provide atomic delivery to all members of the group that do not partition or fail away from the token ring for a sufficient period of time (say 30 seconds). If some sites partition away or fail, a message may have been delivered to these sites but not be delivered to any of the remaining sites. However, if any member of the remaining partition got a packet, all of the remaining sites will also get it, with the ordering guarantees preserved. Ordering guarantees between packets of different QOS levels are determined by the lowest QOS of the packets in question. For example, for a set of packets S1 with source ordered QOS and a set of packets S2 with totally ordered QOS, the best guarantee that is provided over the union of the two sets is source ordering.

ISIS first defined the notion of virtual synchrony[BSS91], [Birman93]. Virtual synchrony often allows a distributed application to execute as if its communication was synchronous, when it is actually asynchronous. The key requirement for virtual synchrony is that all sites see the same set of messages before and after a group membership change. In other words, for a given set of packets delivered to a group, a membership change operation will partition these packets into the

\begin{tabular}{|l|l|l|}
\hline QoS & Name and Service Guarantees & Delivery Time \\
\hline Unreliable & $\begin{array}{l}\text { Packets are delivered } 0,1 \text { or more times, } \\
\text { in any order. }\end{array}$ & Immediately upon receipt of a data packet \\
\hline Unordered & $\begin{array}{l}\text { Packets are delivered at least once, in } \\
\text { any order. }\end{array}$ & $\begin{array}{l}\text { Immediately upon receipt of a data packet, with } \\
\text { missing packets detected and rerequested }\end{array}$ \\
\hline $\begin{array}{l}\text { Source } \\
\text { ordered }\end{array}$ & $\begin{array}{l}\text { Packets are delivered exactly once, in the } \\
\text { order they were sent from each source. }\end{array}$ & $\begin{array}{l}\text { After all of the data packets from the same } \\
\text { source and with smaller sequence numbers } \\
\text { have been delivered }\end{array}$ \\
\hline $\begin{array}{l}\text { Totally } \\
\text { ordered }\end{array}$ & $\begin{array}{l}\text { Source ordered, plus all totally ordered } \\
\text { packets are delivered in the same order at } \\
\text { all sites. }\end{array}$ & $\begin{array}{l}\text { After all of the data packets with smaller } \\
\text { timestamps have been delivered }\end{array}$ \\
\hline K resilient & $\begin{array}{l}\text { Totally ordered, plus delivery is atomic at } \\
\text { all sites that do not fail or partition, } \\
\text { provided that no more than } \mathrm{K} \text { sites fail or } \\
\text { partition at once. }\end{array}$ & $\begin{array}{l}\text { After all of the data packets with smaller } \\
\text { timestamps have been delivered and the token } \\
\text { has been transferred K-1 times }\end{array}$ \\
\hline $\begin{array}{l}\text { Majority } \\
\text { resilient }\end{array}$ & $\begin{array}{l}\mathrm{K} \text { resilient, with } \mathrm{K} \text { set to (MaxN+1)/2, } \\
\text { where MaxN is the highest number of } \\
\text { sites in the token ring for any token list in } \\
\text { the OrderingQ }\end{array}$ & $\begin{array}{l}\text { Same as K-Resilient, but also requires that only } \\
\text { a majority partition can continue functioning. }\end{array}$ \\
\hline $\begin{array}{l}\text { Totally } \\
\text { resilient }\end{array}$ & $\begin{array}{l}\mathrm{K} \text { resilient, with } \mathrm{K} \text { set to } \mathrm{N} . \\
\text { Fing }\end{array}$ & Same as Majority Resilient \\
\hline
\end{tabular}

Figure 2.2: RMP QoS levels 
same two sets at all sites, and all packets in the first set will be delivered at all sites before any packets are delivered in the second set. RMP provides virtual synchrony for packets that have a QOS of at least totally ordered. This is done by implementing each membership change as a packet with a totally ordered QoS.

Total ordering and atomicity of delivery is only guaranteed if no sites fail or partition away from a token ring. To provide guarantees on atomicity and total ordering in the face of failures, higher levels of QaS are provided. These include K-resiliency, majority resiliency, and total resiliency. These guarantees are provided primarily by requiring sites to wait to deliver packets until they have seen that the token has been passed at least $K$ times after they would have normally delivered a packet. Because each site that accepts the token must have all previous packets, by enforcing this delay, RMP ensures that both the current token site and the $\mathrm{K}$ previous token sites have that packet when it is delivered. This allows up to $\mathrm{K}$ sites to crash or partition away and still guarantee atomicity of packet delivery. K-resilient guarantees are particularly useful for applications that operate with a low probability of failure on a network that can't partition, such as a single LAN.

The Totem work[AMSM92] has pointed out that virtual synchrony as defined by ISIS only applies in the case where groups do not partition. When partitions occur, it is possible that the two sets may see a different total order of packet delivery, and packets delivered to a minority partition may not get delivered to a majority partition. To get around this. they have defined another condition, extended virtual synchrony, which they provide. Extended virtual synchrony provides the same guarantees as virtual synchrony, but operates even in the presence of arbitrary group partitions. RMP provides extended virtual synchrony through the use of majority resilience and total resilience. Unlike Totem, RMP requires that only a single partition be allowed to proceed in the case of network partitioning. This is provided by only allowing a partition to continue operation after a failure if it contains at least a majority of the processes that were in the ring. To calculate the number of processes that were in the token ring, the maximum number of processes in any token list that is currently being held for possible retransmission is used. The minimum partition size is specified on a per token ring basis, so that if a given token ring is not using any majority or totally resilient messages, all partitions of it can be allowed to proceed if desired. Majority resilience guarantees that the total ordering of packets is kept consistent between all partitions, and that all of the sites that remain in the majority partition will atomically deliver any packet that has been delivered at any of the sites in the token ring. However, in the face of partitions and failures, the exact set of 
these sites is not known at delivery time. To get around this, Totem defines and provides safe delivery, which guarantees that when a packet is delivered, it has been received by all of the hosts in the current token list, and will be delivered by each of these hosts so long as they do not fail. Unlike majority resilience, safe delivery will deliver a packet at all sites in the current token list irregardless of network parititions. RMP provides this through total resiliency, where $\mathrm{K}$ is always set to the current size of the token ring. After each message has been passed around the entire token ring. all sites must have it and so it can be safely delivered.

\subsection{Communication model}

There are two main options in current communication addressing. Protocols such as TCP and UDP require explicit naming of the destinations of communication, while systems such as Grapevine[BLNS82] and the MessageBus[Carrol193] allow implicit naming through a publisher/subscriber model of communication. RMP supports both approaches. RMP processes usually join or "subscribe" to a group by specifying the name of a token ring to join. and other processes "publish" or send messages to this group by using the token ring name or a token list ID associated with the name. When this model is used, messages sent to the group name are delivered automatically to all RMP processes, if any, that are members of that token ring. so no explicit knowledge of the membership of a group is needed. As explained above. RMP does this by mapping token ring names into [multicast address, port. TTL\} tuples that are used to locate other members of the token ring.

Instead of specifying a token ring by its name, RMP processes may instead unicast a packet to another RMP process (specified by an IP address and a UDP port) that is a member of the token ring and request that this site forward the packet for it. This can both be used to send packets to a token ring from a non-member or non-multicast capable member of that ring, and to join a ring based on a known RMP process ID instead of an IPM address and port. This addressing is similar to the explicit naming used in most point to point protocols. When coupled with the ability of members to query the current membership of a token ring at any time and the feature that members are notified when the ring membership changes. RMP allows processes to exert explicit control over group naming and membership when desired.

In addition to the publisher/subscriber model of group communication, RMP also supports the client-server model of communication by allowing non-members to send to a ring (using either implicit or explicit addressing) and to optionally get replies using a multi-RPC mechanism. When a client sends a packet to the members of a token ring (the servers), it 
can specify the response type it expects. It will usually either request an acknowledgment or a reply, although it can also specify neither or both. An acknowledgment is sent back by a single member of the ring (the current token site) after the packet is delivered to the token ring with the requested ordering, atomicity, and reliability guarantoes. A reply also comes from a single member of the token ring, but is produced by an application using RMP instead of the RMP code itself. A reply will usually contain data that is returned to the client. These multi-RPC packets are asynchronous and have integrated flow and congestion control.

When a packet is sent to a token ring, an optional handler number may be specified for the packet. Handler numbers are used to generate replies to member data packets and to multi-RPC packets. In the current implementation of RMP. there are six different handler numbers per token ring. If one of the RMP processes in the token ring is the handler for that handler number, it will be notified upon delivery of the packet that it is supposed to respond to it. This allows multiple applications that provide a given service to all be in a token ring, and at most one of them will respond to any given request. The handler service is provided through the use of a set of mutually exclusive handler locks. A member of a token ring can request a handler lock, and it will only be granted if no other site currently holds that lock. Once granted, it is the handler for packets sent to the handler number associated with that lock until it releases the handler lock or is removed from the token list due to a failure or a partition. Handler locks can also be used for any other type of service or distributed application that needs mutually exclusive locks. Handler locks are very efficient, needing the equivalent of only a single totally ordered message for each lock operation and each release operation.

\subsection{Non multicast capable processes}

For efficiency. RMP should be run on top of an unreliable multicast service. However, for flexibility, it also supports the use of hosts that are not multicast capable. This is done through the use of forwarding and multicast capable flags. Each RMP process has to have a UDP/IP port open for sending and receiving packets in addition to any IP Multicast addresses it is using. Any packet that is sent to the UDP/IP port for an RMP process can have a forwarding flag turned on. This flag directs the receiving process to copy it to the IP Multicast address for that group, with local loopback disabled.

Some of the packets sent in RMP are unicast to their destinations. As each RMP process is identified by its unicast address, these addresses are already stored in the token list for a token ring. Each of these RMP process IDs in the token 
list contains an additional multicast capable flag denoting whether or not they can receive IP Multicast packets. When a multicast is sent to a token ring, if any members are not multicast capable, the sender must also send a unicast to each of these destinations. In the rest of the paper, wherever we mention a multicast from a group member, we are referring to this extended notion of multicast. Because the case of having all sites be multicast capable can easily be stored as a flag at each site. this common case will pay hardly any penalty for the extra flexibility this service provides.

\section{Algorithms}

RMP provides all of these services through a set of five main algorithms. The token ring algorithm handles the delivery of packets to the members of a token list. When a membership change request or a handler lock request occurs on a token ring, the token list change algorithm creates a new token list and updates it at each member of the ring. When failures occur in a token ring, the reformation algorithm polls the current members of a token ring. synchronizes them to the same point. creates a new token list for that ring, and commits it at each member. Non members can participate in a token ring using the multi-RPC algorithm. Finally, all of the senders in RMP use a flow and congestion control

\begin{tabular}{|l|l|}
\hline Packet Type & Description \\
\hline Data Packet & Contains data to the token ring from a token ring member \\
\hline \multicolumn{2}{|c|}{ Control Packets } \\
\hline ACK & $\begin{array}{l}\text { Provides positive acknowledgment and total ordering for one or more data } \\
\text { packets and/or non-member data packets, as well as passing the token and } \\
\text { confirming that the token has been accepted by the site that sent the ACK }\end{array}$ \\
\hline Confirm & $\begin{array}{l}\text { Provides positive acknowledgment to the last token site that the new token } \\
\text { site has accepted the token. This function is usually performed as part of an } \\
\text { ACK. }\end{array}$ \\
\hline NACK & Requests retransmission of one or more packets \\
\hline New List & $\begin{array}{l}\text { Contains a new token list and its own ACK. It is also used during failure } \\
\text { recovery. }\end{array}$ \\
\hline List Change Request & Requests a change to the current token list \\
\hline \multicolumn{2}{|c|}{ Failure Recovery Packets } \\
\hline Recovery Start & $\begin{array}{l}\text { Sent out when a failure is detected to start the recovery process. Is sent } \\
\text { repeatedly by the initiator until the sites in new token ring are synced to the } \\
\text { same point. }\end{array}$ \\
\hline $\begin{array}{l}\text { Recovery Vote } \\
\text { The response to a recovery start packet, notifying the initiator of the sync } \\
\text { point for this member of the new list }\end{array}$ \\
\hline Recovery ACK New & Acknowledges receipt of the New List packet for the new token list \\
\hline List & Provides notification that an error in the reformation protocol occurred \\
\hline \multicolumn{2}{|c|}{ Non Member Packets } \\
\hline Non Member Data & A data packet from a process which is not a member of the token ring \\
\hline Non Mermber ACK & An ACK or response to a non-member data packet. \\
\hline
\end{tabular}

Figure 3.1: RMP packet types 
algorithm based on the Van Jacobson TCP congestion control algorithms.

\subsection{Packet types}

RMP uses ten packet types in its communication. These are listed in figure 3.1. The token ring algorithm uses Data packets, ACKs, Confirm packets. and NACKs. The token list change algorithm uses New List and List Change Request packets. The recovery algorithm uses four recovery packet types as well as New List packets. The multi-RPC algorithm uses Non Member Data packets and Non Member ACK packets. Flow control and congestion control is based on ACK packets and NACK packets.

\subsection{Token ring algorithm}

The biggest decision in building a reliable multicast protocol is how to guarantee reliability. Traditional protocols use positive acknowledgments (ACKs) from the destination to acknowledge successful receipt of a packet. This approach does not scale well to a multicast system. because each destination has to send an ACK for each packet or set of packets. This largely defeats the advantage of using multicast packets, because it decreases both the efficiency and the performance of the protocol. Even though these acknowledgments are small, because they all are sent at the same time they can cause network congestion. In addition, having to process an ACK from each destination increases the load on the sender and decreases the performance of the protocol. To get around this. many systems use negative acknowledgments (NACKs). Negative acknowledgments shift the burden of error detection from the source to the destinations. Packets are stamped with sequential sequence numbers which destinations use to provide reliable delivery by detecting gaps in the sequence numbers and requesting retransmission of the packets corresponding to the gaps. Because the information that a packet has been received is never propagated back to the sender, the senders in these protocols do not ever know for certain that a destination has received a packet. Because of this, senders have to indefinitely keep a copy of each packet sent if the protocol is to be considered truly reliable. In addition, a lost packet will not be detected until another packet is received successfully, which may take a long time if the packet is the last to be sent to the ring for a while. Because of these problems, the token ring algorithm uses a combination of these two approaches. This algorithm is a modified version of the work originally done by Chang and Maxemchuk[ChMa84], [ChMa83]. 
In RMP, all data packets are stamped with a tuple [RMP process D, sequence number for that process, QWS level for the packet\} which uniquely identifies each data packet. Data packets are multicast to the members of the token ring and are handled by a primary receiver called the token site. When the token site receives one or more data packets, it multicasts a positive ACK out to the members of the token ring. In certain cases, an ACK may be sent out that doesn't acknowledge any data packets (see below). Each ACK contains zero or more identifying tuples for Data packets. along with a global sequence number, called a timestamp, which serializes all of the ACK. Data, and New List packets in a token ring. For a given ACK, the ACK is given the value of the timestamp, and each data packet ordered by the ACK is given a consecutive timestamp. For example, an ACK that orders two data packets might have a timestamp of 8 . In this case, the first data packet would receive timestamp 9 and the second would be numbered 10 .

Each ACK performs a number of functions:

-It lets the sender know that the current token site has received the packet. In this way it functions as a traditional positive acknowledgment to the sender.

-The timestamps in the ACKs provide a total and causal ordering on messages.

-The timestamps also provide a global basis for the detection of dropped packets. The receivers can detect any missed packets, both ACKs, Data, and New List packets, through these global sequence numbers. With multiple simultaneous senders. this provides for faster detection of lost packets than does detection based on sequence numbers from each sender.

As with other NACK based solutions, this does not solve the problem of guaranteeing the detection of dropped packets by the destinations and the corresponding problem of unlimited buffer space. To solve this problem, the token site is passed among all the processes in the token ring. Along with the other functionality of an ACK. each ACK also serves to pass the token to the next member of the ring. Not only does rotating the token balance the load of the ACKs between the sites, it also solves the buffer problem. Given $\mathrm{N}$ members of a ring, once the token has been rotated $\mathrm{N}$ times, the token site knows that all messages with a timestamp at least $\mathrm{N}$ smaller than the current timestamp have been received at all destinations, and so the token site no longer needs to store these messages for retransmission.

To pass the token, a field in each ACK names the current token site that issued the ACK and the new token site. When a RMP process receives an ACK naming it as the new token site, it checks to see if it has received all of the packets 
with timestamps up to that of the last packet ordered by this ACK, if any. If not, it uses NACKs to request them from the previous token site. Once the process has all the packets, it declares itself to be the new token site. Passing the token requires positive acknowledgment, so the new token site must let the old token site know that it has accepted the token by either multicasting out a new ACK, or by sending the old site a unicast Confirm packet.

Changes in the token list are requested by a process with a List Change Request packet, which is handled similarly to a data packet. However, instead of sending an ACK on a List Change Request packet, the token site processes the change and sends out a New List packet. A New List packet serves as both a data packet containing information for the token ring and as its own ACK.

Ordering of packets. detection of missing packets, and buffering of packets for retransmission is all handled with the use of two lists, the Datalist and the OrderingQ. The DataList contains Data and List Change Request packets that have not yet been ordered. The Ordering $Q$ contains slots, each of which holds a pointer to a packet, the delivery status of the packet (Missing, Requested. Received, or Delivered) the \{source, sequence number, QoS\} identifying tuple for the packet. and the timestamp for the packet. The fields in a slot are not all used at all times. The slots in the OrderingQ always have monotonically increasing timestamps.

When a Data or List Change Request packet is received, it is placed into the DataList. When an ACK or a New List packet is received. it is placed in the OrderingQ. creating one or more slots on the end of the queue if necessary. List Change Request packets are never placed into the OrderingQ, as they are transformed into New List packets when they are ordered. Each packet occupies exactly one slot in the OrderingQ. When an ACK is placed in a slot, the tuples it contains identifying data packets are copied to the slots immediately succeeding it, creating new slots if necessary.

Whenever a Data or ACK packet is received, the OrderingQ is scanned through once to match up Data packets in the DataList with empty slots that have been created by an ACK. When a slot is found that has the same identifying tuple as a Data packet in the DataList, the packet is moved from the DataList to that slot. When holes occur in the OrderingQ. NACK packets are sent out, requesting retransmission of these packets. The exact policy for determining the destination of the NACKs and whether or not the retransmissions should be unicast or multicast is a topic for continuing research. The default policy is to send NACKs either to the sender of the packet, if known, or to multicast them to the token ring and name the last known token site as the site to handle them. If there is no response to a NACK within the specified 
timeout, a site will resend the NACK to a different site. This will continue up to a constant threshold, after which point the NACK will be multicast to the entire ring for any site to respond to.

A site is not allowed to accept the token until there are no empty slots in the Ordering $Q$ up to the last data packet ordered by the ACK naming this site as the new token site. Because of this, the OrderingQ does not need to contain any more than N ACKs and New List packets, where $\mathrm{N}$ is the current number of sites in the token ring. When there are more than N ACKs and New List packets in the OrderingQ, the slots at the front of the queue and their corresponding packets are dequeued and freed until this condition is met.

In addition to bounding the buffer space needed in a ring, passing the token guarantees that site failures and dropped messages are detected within $\mathrm{N}$ messages. In order to bound the amount of time before a lost packet or a failed site is detected, RMP sends NULL ACKs, which pass the token but do not order any packets, if the token site does not receive a message within a given period of time. With RMP, this is currently on the order of 1 second. When a ring goes quiescent for an extended period of time, the token is passed all the way around the ring once and then stops. At this point, all of the sites are guaranteed to have all of the messages.

If a site repeatedly fails to receive the proper response to one of the actions that requires a positive acknowledgment, it declares the site dead and runs the reformation protocol. described below in section 3.4.

\subsection{Token list changes}

A new token list is created whenever a RMP process joins a token ring, leaves a token ring, is granted a bandler lock for a token ring, or releases a lock for a token ring. Sites that fail or partition away are handled by the fault recovery algorithm, described below. For the other list changes, a site requests the change by multicasting an unreliable List Change Request packet to the group. List Change Request packets require positive acknowledgment, and are resent periodically until this is received or a fault in the ring is declared. The current token site serializes these change requests and sends out New List packets in response.

List Change Request packets are handled similarly to Data packets. except that when the token site would normally send an ACK packet to order and acknowledge a Data packet, it instead generates a New List packet. When a token site creates a New List packet, it makes the requested change to the current token list, if possible, and puts this list into the second half of the New List packet. It fills the first half of the New List packet with the fields of an ACK, including a 
timestamp ordering the New List packet and the ID of the next token site. In this way, a New List packet acts as both a totally ordered Data packet whose destination is RMP instead of an application, and as its own ACK. When a New List packet is received, it is put on the OrderingQ, and then broken down into its two halves. The ACK half is processed first. which will put the New List packet into the OrderingQ. From this point, it is handled like a data packet. When the data half of the New List packet would normally be delivered to the RMP process, it is instead committed and a notification of the token list change is delivered to the application.

When a RMP process is joining a token ring, it can send the List Change Request packet to either the IP Multicast address and port for a token ring, or it can send it to the IP address and port of a known member of a token ring. In the latter case, the process sets a forwarding flag on the packet. and when the packet is received it is forwarded to the token ring. The joining member will repeatedly send the List Change Request packet until it receives a New List packet naming it as the new token site. Because the joining process may not yet know the IP Multicast address for the token ring, this New List packet is also unicast to the joining process's UDP address and port, and it contains the multicast address and port for the token ring. A new member is always added in to the token list directly after the current token site. This forces the new member to take an immediate role in the token ring, providing positive acknowledgment that it has joined the ring and started processing messages at the correct time. If no New List packet is received after a certain number of retries to a token ring, the new RMP process creates a new token ring with only itself in it. If a ring does actually exist for that token ring name and multicast address, but is currently unreachable due to a network partition, a second ring may form. Because token ring IDs are unique, even if this partition heals, the rings will never overlap or merge.

When a site removes itself from a ring, it must remain a member of the token ring until after it has seen and committed the new token list removing it from the ring. After leaving the ring, it must continue to process NACK requests and keep track of token passes until it no longer has any packets from the old list that it must hold for retransmission. A packet must be held for retransmission be an exiting site while its resiliency level is greater than or equal to the number of times it knows that the token has been passed since it last held the token. 
List Change Request packets requesting a handler lock will not be granted if another process already holds that handler lock when the token list processes the request. If a request is denied, a New List packet is still generated, but the token list it contains will be the same one the token ring was using before.

\subsection{Fault recovery}

There are four types of packets in the normal mode of the RMP protocol which require positive acknowledgmentData packets, ACKs, List Change Requests, and NACKs. If at any time a RMP process repeatedly times out and resends one of these packets more than a set number of times (ten in the current implementation) it decides that a failure has occurred and starts the fault recovery protocol. The initiator of the fault recovery protocol repeatedly polls the sites in the token ring to determine who is still active and reachable. generates a new token list. makes sure that all sites in the new token list have the same set of packets in their OrderingQ, and then commits this list at all sites in the new list using a two phase commit protocol. Because token passes are one of the actions that require positive acknowledgment. a failure will be detected within $\mathrm{N}$ token passes.

The fault recovery protocol is broken into two halves--creating and synchronizing the list, and committing the list. In the first half, the initiator repeatedly queries the other sites in the old token list to see if they are up, to see what the highest version of token list they have seen is, and to find out what their current sync point is. The sync point for a RMP process is defined as the highest consecutive timestamp it has in its OrderingQ plus the highest consecutive sequence number it has received from each site in the old list. This information corresponds to the timestamp of the highest packet that a RMP process has delivered with a QOS of at least totally ordered concatenated with the highest sequence number of a packet delivered with a QOS of at least source ordered from each RMP process in the ring. By responding to a query, a site provides the requested information and confirms that it has joined the new token list the initiator is creating. A process is only allowed to join a token list with a larger version number than any it has seen before, and it is only allowed to join one list at a time. If any of these conditions are not met, each process detecting the error multicasts an abort reformation packet out, aborts its own reformation, and waits for a random timeout period or another reformation start packet from another site before restarting.

The goal of the first phase is to have as many of the old sites as possible join the same new token list and reach the same sync point. If some of the sites are missing packets, this may be an iterative process. If the initiator receives a 
higher sync point from another process than the one it has, it stores this as the new sync point for the list and forwards this sync point to the other processes. All sites that are missing packets request those packets and send higher sync points as they receive them. After either all but one of the old sites in the token list have responded and been brought to the same sync point, or after no further progress has been made towards the shared sync point after a set number of retries. the initiator creates a new list with all of the members of the old list that have reached the same sync point. If the initiator receives a packet from another initiator with a smaller or equal version number, it sends back a packet notifying that initiator that it should abort its reformation. If the initiator receives a packet from another initiator with a larger version number or if it is not itself able to reach the shared sync point, it sends out an abort packet to the members of the list and aborts its own reformation process, setting a random timeout before it starts the reformation again.

Once the initiator has created a new list, it must check that this new list has at least the minimum number of sites specified in the old version of the token list. When joining a token list, each site specifies the minimum number of sites that must remain in a partition in the case of a failure. The minimum size for the new token ring is the maximum of these values for each member in the old ring. This value can be either a constant, a symbolic constant specifying the majority of the members of the old list, or a constant for all of the members in the old list. In the latter case, any failure will always cause the ring to stop operation.

If this test is passed, the initiator creates the new list and must commit it at all of the sites. First, it multicasts a New List packet to all of the members of the new list and requests a reply from each of them. Once it has received these acknowledgments from each member, it commits the new list itself and makes itself the new token site. If it has any Data packets waiting to be acknowledged, it sends out an ACK on these packets. Otherwise, it sends a NULL ACK to pass the token. After each of the members of the new list receive this or any other ACK to the new list. they also commit the new list and start processing packets as normal.

\subsection{Multi-RPC delivery}

The client server model of communication has become widely accepted as a powerful way of providing services to users. While RMP could support this model simply by having all clients and servers join a token ring, this is often inefficient and will limit the scalability of client/server groups. As an alternative, RMP provides facilities for RMP processes that are not members of a token ring to use a multi-RPC algorithm to send data to a ring and to receive 
acknowledgments of successful delivery and/or responses from a handler. This is a powerful feature, for it allows multiple servers to exist in a token ring, and all of them can get messages from clients. These messages can be automatically acknowledged, or a single member of the token ring can be selected to handle the request and reply to it. Because these Non Member Data packets can be delivered with all of the QoS levels of a Data packet sent from a token ring member, multi-RPC packets can be delivered with all of the reliability, ordering and atomicity guarantees of a member data packet.

The two main changes between a Data packet and a Non Member Data packet are that a Non Member Data packet can include a token ring name instead of a token list ID and it includes two flags that aid in getting acknowledgments or responses. Since a non member will often not know the current token list ID for a token ring. it can instead specify the textual representation for the ring. When it gets a reply back from the token ring, it can cache the token list ID included in this reply and use this in subsequent Non Member Data packets. The first flag specifies whether or not an acknowledgment should be sent in response to the Non Member Data packet. If so, then when the current token site delivers the Non Member Data packet to its application, it unicasts a Non Member ACK to the sender. Because the sender has the responsibility for making sure the packet is delivered reliably, this acknowledgement may need to be sent to the sender multiple times. This is done by sending another copy of the Non Member ACK each time a duplicate of a Non Member Data packet that has already been delivered is received. The second flag provides the same repeat reply functionality but for replies instead of acknowledgements. When this flag is turned on, the Non Member Data packet will be delivered multiple times to the application. This is usually used in conjunction with a handler number for the packet. If a handler number is specified, the member of the token ring that holds that handler lock will be responsible for replying to the Non Member Data packet. This reply will usually be used as the ACK for this multi-RPC call, and must be sent as many times as necessary until the non member receives it.

In order to provide source ordered delivery guarantees on non member packets, the members of a token ring have to keep track of the highest delivered sequence number from each non-member. These are stored in the token list along with the sequence numbers for members, but are marked with a non-member flag. Because of this, they are sent out as part of each New List packet, and each joining member will receive a copy of these sequence numbers. It is the responsibility of a non member that is sending source ordered packets to set a flag on the first packet sent to a token ring. 
notifying the destinations that it is the first packet. This notifies the members that they can create a sequence number for this process starting with this packet. Optionally, these non-member sequence numbers may be flushed if no packet has been received from that site within two times the maximum TTL of a packet in the internetwork. If this policy is used, then non-member senders must keep track of when their sequence numbers may have been flushed out and reset the first packet flag when this occurs.

\subsection{Flow and congestion control}

Flow and congestion control policies for reliable multicast protocols are an open problem. Because reliable multicast protocols primarily use NACKs for error detection, there is no existing explicit feedback path with which destinations can signal losses or low buffer space to the senders. In addition, the throughput for a multicast group should be divided up between the members of the group who are trying to send, but the policy for this division is usually dynamic and not known in advance. Because of this, the flow and congestion control policies used by RMP are designed to be orthogonal to the rest of the protocol. Flow and congestion control policies can be inserted easily into the protocol, and different policies can be used in different environments. As the default, we propose a modified sliding window protocol based on the Van Jacobson algorithms used in TCP [Jacobson88].

Two of the most common schemes used today for flow control and congestion control are sliding windows and leaky buckets. Leaky bucket schemes, which enforce explicit rate controls on each sender, are classified as predictive controls. They try to predict how much bandwidth each sender can use at any given time, and then mandate that the senders do not exceed this. Calculating the values for these rates is difficult, and they must divide up the bandwidth between the senders on a relatively static basis. This decreases the flexibility and throughput attainable by these schemes. In addition. it is difficult with a leaky bucket scheme for the destinations to signal back that their buffers have been overrun if a destination

process stalls for some reason. For the new very high speed WAN networks that are being proposed, the cost of congestion can be very high because a sender can have hundreds of packets in transit at once. For these networks, a leaky bucket or rate control scheme may be necessary.

For networks that have a lower latency bandwidth product, the drawbacks of a leaky bucket scheme may not be necessary. For RMP, we propose an adaptive flow and congestion control scheme based on a modified sliding window scheme. This algorithm treats flow control and congestion control as the same problem and solves it in part by using 
some of the algorithms proposed by Van Jacobson for TCP. Each sender maintains a window of how many bytes it can have in transit at once. When a packet is sent, the window size is decreased by the number of bytes sent, and when the first copy of an ACK for a packet is received the window size is increased by the same amount. This causes the flow control feedback to be rotated among members. If a site is overrun and runs out of buffers, when it gets the token site it can delay acknowledging any more packets until it can process incoming packets again. If the RMP process delays too long, however, it will be considered to be faulty, and it will be removed from the token ring. This is usually desirable behavior, as otherwise the other members of the ring will block indefinitely on this one site.

The bulk of the flow and congestion control is provided by controlling the maximum size of the window at each sender. The maximum size of this window grows according to the slow start algorithm proposed by Van Jacobson, and is decreased when an expired retransmission alarm occurs. The Van Jacobson algorithms were originally designed to just provide congestion control. However, because they provide such good adaptive congestion control, RMP also uses them for additional flow control by treating NACKs as another signal of congestion.

The Van Jacobson algorithms for congestion control that are used by RMP include:

(1) round-trip-time variance estimation

(2) slow start

(3) dynamic window sizing on congestion

(4) exponential retransmit timer backoff

Round-trip-time variance estimation comes from the observation that when a network path becomes congested, the variance on packet latency becomes very high compared with the average. "If the network is running at $75 \%$ of capacity...one should expect the round-trip-time to vary by a factor of 16."[Jacob88] The proposed algorithm continually estimates this variance, and eliminates most of the spurious retransmissions while still maintaining timeouts small enough to detect dropped packets quickly.

The slow start algorithm (2) is used to increase the window size from 1 packet to the maximum window size that the receiver allows that does not cause congestion, as calculated by algorithm (3). This is done by incrementing the window size by one packet each time that an ACK is received. Because the window size is constantly growing, slow-start actually increases the window size fairly quickly. It will increase from 1 to $\mathrm{W}$ on a network with latency $L$ in $L \log _{2} W$ time. 
With the assertion that the timer algorithm almost completely avoids retransmissions that are not due to lost packets and with the observation that most lost packets are due to congestion instead of errors, it follows that most expired timers signal congestion. Algorithm (3) uses this to respond aggressively to this congestion by exponentially reducing the window size by a constant number (currently 50\%) each time that a timer expires. The original protocol actually uses a two-level bound on this window in the face of congestion. It reduces the window size to one packet any time an error occurs, uses slow start to quickly build up to $50 \%$ of the level before the error, and then uses a slower linear increase to build up from there. RMP only reduces the current window size by $50 \%$ because RMP modifies the packet lengths according to the window size. The algorithm used for this causes slow start to reach a window size $\mathrm{W}$ (assuming $\mathrm{W}$ is less than two times the maximum packet size) in $\mathrm{O}(\mathrm{W})$ time instead of $\mathrm{O}(\log W)$ time, and this makes the cost of reducing the window all the way to 1 packet too high.

Finally, the exponential retransmit timer backoff is used to double the timer each time it expires, resetting it to the value calculated by (1) when an ACK is finally received. Both this and algorithm (1) are applied to the timers for all of the packets that require positive acknowledgment. Along with further decreasing congestion, this provides an efficient detection method for failed sites. The maximum value for a timer is clamped at a certain value (currently 2 seconds). Then up to $\mathrm{N}$ retransmissions (currently 10 ) are allowed before a site is declared dead. With the currently implemented values this policy detects failures in nearby sites within 5 seconds, and distant sites within $15-20$ seconds.

By using NACKs as signals of dropped packets. these algorithms also provide effective flow control. If a destination gets overnu by the senders, it will drop one or more packets. This will usually be detected by the destination very rapidly. When this occurs, the destination either unicasts a NACK to the sender or multicasts a NACK back to the group. In addition to requesting a retransmission of the packet from the current token site. this NACK aiso informs the original sender (who is named in the NACK) that a destination has lost a packet from that sender. This is treated the same as an expired timer due to a lost packet. and causes the sender to decrease its window size by $50 \%$. In order to make sure that multiple NACKs on the same packet do not each decrease the window size, a cache of the sequence numbers of the last three messages sent by this site that were dropped by another site is maintained by the sender. Incoming NACKs are compared against this cache, and the window size is only modified if a NACK for this message isn't in the cache. 
I/ Avoid "Silly Window" effect

if (A < MIN_PACKET \& $A<W$ )

Delay sending packet until an $A C K$ is received

/ Send up to 1/2 of the window at a time

$S=\min (P, W / 2)$;

// Send at least MIN_PACKET bytes

$S=\max (S$, MIN_PACKET);

// Can only send up to $A$ bytes

$S=\min (S, A)$;

// Reduce effect of lost packets

$S=\min \left(S, M A X \_P A C K E T\right)$;

Fgure 3.2: Packet Size Algorithm
A problem that RMP faces with flow and congestion control is that the rotating token site introduces a higher overhead per acknowledgment than traditional protocols such as TCP. This is compounded by the protocol being more complicated than TCP and thus requiring more processing per packet. To solve this problem, RMP uses larger packet sizes than does TCP. In an error free environment, having the IP or IP Multicasting layer do the fragmentation and reassembly is more efficient than having RMP do it. If

errors occur, the window size quickly drops to a single minimum size packet. The algorithm to determine the size of the packet to be sent out (S), given the current window size (W). the available space in the window (A), and the offered packet size (P), is shown in figure 3.2. The critical step in this algorithm is that up to half of the available window is sent at a time until the maximum packet size has been reached. This trades off a small amount of network utilization in the case of errors for typically higher efficiency of handling packets and higher throughput.

\subsection{Implementation options}

RMP is designed to be implementable in either user-space or kernel space. There are numerous pros and cons to each approach, so RMP does not enforce either approach. Traditional protocols have been implemented as monolithic entities in the kernel. This is motivated primarily out of concerns for security and performance. However, as pointed out by [JHC94], [MaBe93], and [TNML93], user-space implementations can be more easily modified and customized, are easier to debug and experiment with. are more easily ported between different platforms, and can increase the performance of protocols when run on a multiprocessor. In addition. [TNML93] shows that it is possible to implement protocols in userspace without major throughput penalties.

Numerous "software buses" have been implemented recently, which are designed to make applications which do group communication much easier to program. RMP was originally designed as the transport layer for one of these buses, the MessageBus[Carrol193], and supports these types of systems very well. Some of the features that these systems provide on top of RMP are translation of raw data into different formats and objects, filtering of packets based on another level of group or domain identifier, dynamic loading of processes that are requested to provide a service, buffering multiple small 
packets into a single large packet under heavy load, and a uniform communication interface independent of the actual transport used. Other instances of these software buses are Polylith [Purtilo85] and MultiBus [CaMo94].

\section{Performance}

Note to reviewers: We are finishing up the implementation of the second version right now, and should have performance numbers for it over both LANs and WANs by the time this paper is due for publication.

In normal situations with low error rates and moderate to high traffic. TRP requires very close to 2 broadcasts per message. As the traffic rate decreases, this increases to 2 broadcasts plus a unicast due to the confirm or empty ACK packets required to confirm the transfer of the token. As the error rate increases, the number of packets sent increases. but it is always lower than that required with positive acknowledgments for groups of three or more sites including the sender [ChMa84].

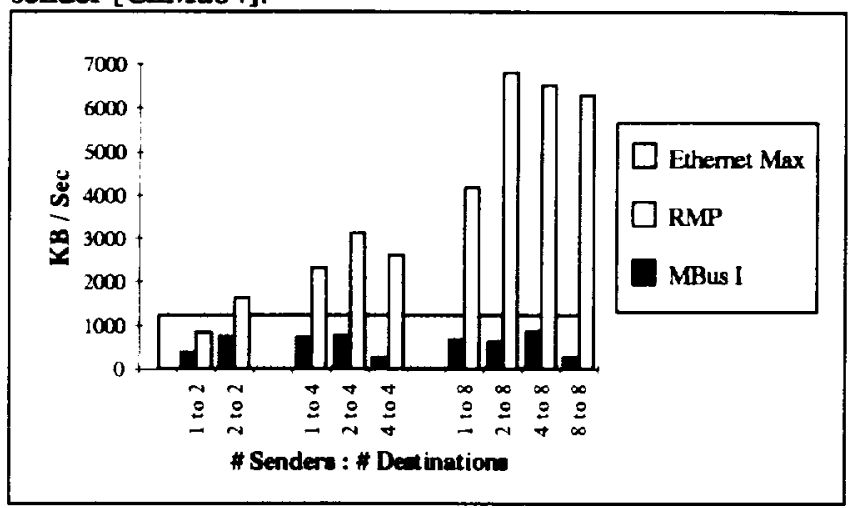

Figure 4.1: Aggregate Throughput (KB/sec)
We measured the performance of the first version of RMP. The algorithms that affect performance in this version are very similar to those used in the version described in this paper, and so should have high applicability. Performance testing was done on 8 SparcStation10's (Sun4m machines) on a lightly loaded 10 $\mathrm{Mb} / \mathrm{sec}$ Ethernet. All of the machines were running SunOS

version 4.1.3. All tests were for totally ordered packets. Throughput was measured by timing the transfer of a $5 \mathrm{MB}$ data file, and so does not include packet headers. Latency was measured by timing the sending of 10,000 minimum length messages (of 64 bytes, including all headers) with buffering and windowed flow control disabled. This causes the protocol to revert to a stop-and-wait acknowledgment system, in which case latency is equal to one over the number of messages sent per second. To eliminate the start up effects of the adaptive time-outs, an initial run was made in each test and discarded. Three runs were made in each test and the results were averaged together. While we do not show the standard deviations, they were quite small.

Figure 4.1 shows the aggregate throughput of the network as a function of the number of sources and destinations. Aggregate throughput is the throughput the user sees. It is computed by taking the amount of data sent from all of the 
senders and multiplying it by the number of destinations. In these tests, the sender is also a destination. This is done so that it can see its own messages totally ordered with the others. This is the way that most groups use totally ordered multicast, and is actually the worst case for the throughput of the protocol because of the increased CPU load of the senders. A single-server. TCP/IP based system, the MBusI, is shown for comparison. The MBusI accepts a TCP/IP stream from each communication client and routes packets between them. The maximum bandwidth of the Ethernet used in this study is also shown. For the case of 2 sources and 8 sinks, RMP achieved an aggregate throughput of 6810 $\mathrm{KB} / \mathrm{sec}$. This is 5.45 times the bandwidth of the Ethernet. It is impossible for any solution that does not use either multicast or broadcast to achieve any result that breaks the Ethernet throughput boundary this way.

In graph 4.2. we see the single sender throughput plotted against the number of destinations. The single sender throughput is equal to the aggregate throughput divided by the number of destinations. To be compatible with other published figures, in these tests the source was separate from the destinations. Data from the MBusI is included for comparison. The performance of all applications (such as UDP ISIS, Sun ToolTalk, the MBusI, and RPC) that do not use hardware broadcast or multicast drops off as a factor of $1 / \mathrm{N}$. The graph is plotted with a logarithmic axis for throughput to better show this limitation. which is a fundamental limit of the network. In contrast. RMP stays roughly constant regardless of the number of destinations. RMP breaks the fundamental unicast limit for two destinations, and so has higher throughput in this environment for all groups of more than one destination. We have not included any numbers from other reliable multicast protocols because no fair comparisons on the same platforms have yet been made.

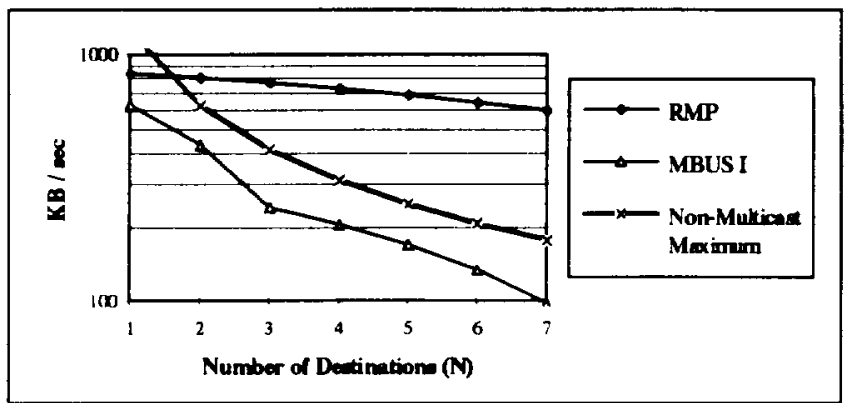

Flgure 4.2: Single sender throughput $(\log \operatorname{scal} \theta)$
In figure 4.3. we see the same factors, but with latency as the metric instead of throughput. Here again, the performance of RMP stays almost constant. While we have not yet been able to make fair comparisons to other protocols, the data that we have shows that the latency of protocols that do not take advantage of hardware

multicast also scales linearly as a function of $\mathrm{N}$ [BiC194]. Note that this graph shows the case when fault tolerance is disabled and $\mathrm{K}=0$. The resiliency factor $\mathrm{K}$ does not affect throughput, but does increase latency. As $\mathrm{K}$ increases, the 
latency under heavy load will increase by a factor of roughly $(\mathrm{K}+2) 2$. While not yet tested, we expect the RMP packets with source ordering to provide lower latency to multiple destinations than unicast systems such as RPC and TCP.

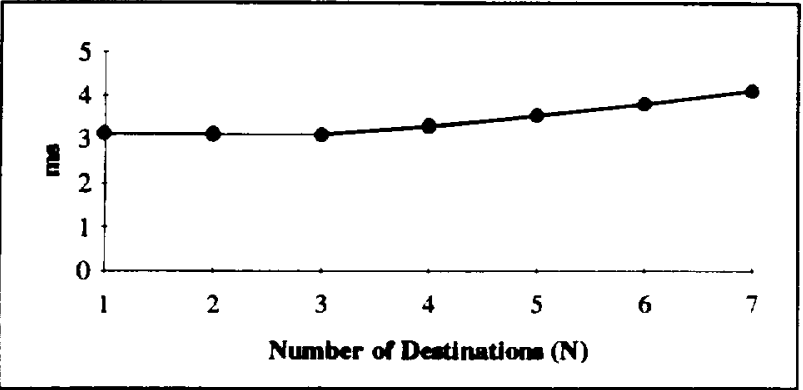

Figure 4.3: Single sender latency with $\mathrm{K}=0$ (ms)

\section{Comparison to previous work}

Work as early as the $\mathrm{V}$ system [ChZw85] implements multicast communication between groups. The V system only implements "best effart" delivery semantics, and does not provide any ordering guarantees on messages.

The MBusI [Carroll93] was the original motivation for RMP. It provides a central server through which clients connect with TCP/IP streams. and an easy to use interface designed to ease the implementation of CSCW applications. It provides both total ordering of messages and reliable multicast, but has very limited scalability.

The Totem protocol [AMSM92] is perhaps closest to RMP in its approach, and has reported similar throughput levels as RMP under heavy load. Totem is perhaps the only reliable multicast protocol that allows consistent total ordering to proceed across multiple partitions of a group. It also uses a token ring approach, but only provides for a single ring for each broadcast domain. Totem avoids using any ACKs by only allowing the token holder to send data out and by having each packet automatically pass the token. This provides high throughput under high load over a low latency network. but provides longer latency under low and asymmetrical loads. In addition, because it only allows a single sender to transmit at a time it will provide lower throughput over longer latency networks. To alleviate this problem they provide gateways to link multiple broadcast domains together.

The ISIS system [BSS91]. [Birman93] is one of the pioneering protocols in this field. It provides causal ordering and, if desired, total ordering of messages on top of a reliable multicast protocol. The reliable multicast protocol requires separate acknowledgments from each destination. which limits performance. A new system that provides causal ordering on top of IP Multicasting has been implemented which is much more efficient than the old system [Clark94], and we hope to compare RMP and this new protocol soon.

The Psync protocol [PBS89] is an ingenious protocol that uses piggybacked ACKs to provide causal ordering of messages and detection of dropped packets. However, both it and the similar Trans [MSMA90] and Lansis [ADKM93] 
protocols require that all of the members of the group regularly transmit messages. The Trans protocol and the ToTo [DKM] protocol implemented on top of Lansis both provide total ordering of messages. These algorithms require that at least a majority of the group members be heard from before a message can be delivered. which causes latency to increase by at least an order of magnitude. For example. for the ToTo protocol to send to a group of 8 destinations under heavy, periodic load from all sources (the best case), the latency is $23.8 \mathrm{~ms}$. This increases to $114.1 \mathrm{~ms}$ for lightly loaded poisson sources.

The Multicast Transport Protocol (MTP) [AFM92] is an example of an asymmetric reliable multicast protocol. One site is the communication master which grants "tokens" to group members to allow them to send data. These tokens provide both flow control and total ordering of messages. This causes over dependency on the master, which limits both reliability and performance. MTP also relies exclusively on NACKs for error recovery, which limits reliability and requires extreme amounts of buffer space.

The protocol by Crowcroft and Paliwoda [CrPa88] is one of the first protocols to propose reliable multicast over an internetwork which supports hardware multicast. The protocol provides different levels of reliability guarantees, and uses positive acknowledgments from all destinations for reliability. The paper analyzes the flooding problems that occur with simultaneous ACKs from many destinations and proposes a windowed flow control system, in some ways similar to that used in RMP, to alleviate these problems.

The protocol by Navaratnam. Chanson, and Neufeld [NCN88] is a centralized multicast protocol that uses a single token site to provide total ordering and reliability. It requires that each site send back a positive acknowledgment before the next packet can be sent. An implementation on top of the V-system takes $24.8 \mathrm{~ms}$ to send a multicast to four destinations. This protocol also is limited in reliability and scalability by the central server. The xAmp protocol [RoVe92] is distributed but also waits for ACKs from all destinations, and so will exhibit performance similar to NCN and ISIS.

The broadcast protocol proposed by Kaashoek et. al. [KTHB88] uses a central token site to serialize messages and NACKs for retransmissions. It piggybacks ACKs onto sent messages and has the token site regularly contact silent sites in order to limit buffer space. This protocol has reported very good latency (as low as $1.3 \mathrm{~ms}$ for a NULL packet) because it has been implemented on top of bare hardware. However, because each message must be transmitted twice it will 
fundamentally achieve lower throughput than RMP .- $600 \mathrm{~KB} / \mathrm{sec}$ is a rough upper bound for a $1250 \mathrm{~KB} / \mathrm{sec}$ Ethernet, as compared to $842 \mathrm{~KB} / \mathrm{sec}$ for RMP. This will also limit the latency for larger messages; as a $8 \mathrm{~KB}$ packet in their protocol will spend a minimum of $13.1 \mathrm{~ms}$ on the Ethernet, as opposed to $6.7 \mathrm{~ms}$ for the message and ACK of RMP.

\section{Conclusions}

In this paper we have described the basic mechanisms and algorithms of RMP, a fully distributed, totally ordered, reliable, atomic, K-resilient fault tolerant multicast protocol. We have shown that RMP provides these features with very high performance. In a LAN, RMP provides much higher throughput to groups of two or more destinations than any protocol that does not take advantage of multicast or broadcast. In addition. it provides lower latency to groups of 3 or more destinations than most other protocols. Much work has gone into providing reliable multicast services with lower ordering guarantees because it was believed that the performance of a totally ordered multicast protocol was inherently low. RMP suggests that this is not the case, and that an efficient reliable multicast service can provide total ordering of messages for only a small latency penalty. Finally, because of its use of multiple token rings, an optional client/server architecture, its fully distributed nature, and its flow and congestion control algorithms, we expect RMP to scale gracefully and efficiently to large groups spread over a large internetwork, and we plan to test this hypothesis in the near future.

\section{Acknowledgments}

This work is supported in part by the NSF through grants CCR-9007195 and CCR-9108931, by NASA through cooperative research agreement NCCW-0040 and grant NAG 5-2129, by the NASA headquarters office of safety and mission assurance (OMSA), by the US Army Corps of Engineers, and by Sun Microsystems, Intel, Bull, Hewlett-Packard, DEC and Fujitsu/Open Systems Solutions. In addition, we would like to thank Alan Carroll for his work on the MBusI which inspired this whole project, Doug Bogia and Bill Tolone for their constant support, and Nick Maxemchuk and JoMei Chang, whose work defined so much of RMP.

\section{References}

[ADKM91] Y. Amir, D. Dolev, S. Kramer and D. Malki. "Transis: A Communication Sub-system for High Availability." Technical Report CS9113, Hebrew University of Jerusalem, Nov. 1991.

[AFM92] S. Armstrong, A. Freier, K. Marzullo. "Multicast Transport Protocol". RFC1301. (February, 1992). 
[AMSM92] D. A. Agarwal, P. M. Melliar-Smith, and L. E. Moser. "Totem: A protocol for message ordering in a wide-area network." Proceedings of the First ISMM International Conference on Computer Communications and Networks (San Diego, CA. June 1992). pp. 1-5.

[ARP93] R. Aiello, E. Pagani, G. P. Rossi, "Design of a Reliable Multicast Protocol". Proceedings of IEEE INFOCOM '93 (San Francisco, March 1993). pp. 75-81.

[APR93] R. Aiello, G. P. Rossi, E. Pagani. "Casual Ordering in Reliable Group Communications." Proceedings of ACM SIGCOMM '93 (San Francisco, Sept. 1993). pp. 106-115.

[BLNS82] A. Birrell, R. Levin, R. Needham, M. Schroeder. "Grapevine: An exercise in distributed computing." Communications of the ACM, 25, 4, April 1982. pp. 260-274.

[BSS91] K. Birman, A. Schiper. P. Stephenson. "Lightweight Causal and Atomic Group Multicast." ACM Transactions on Computer Systems. 9, 3 (Aug. 1991). pp. 272-314

[BiCl94] K. Birman, T. Clark. "Performance of the Isis Distributed Computing Toolkit" Technical Report TR-94-1432, Dept. of Computer Science, Cornell University.

[Birman93] K. Birman. "The Process Group Approach to Reliable Distributed Computing." Communications of the ACM. December, 1993, 36,12. pp. 37-53.

[CaMo94] J. Callahan, T. Montgomery. "A Decentralized Software Bus based on IP Multicasting." Proceedings of Third Workshop on Enabling Technologies: Infrastructure For Collaborative Enterprises, Morgantown, WV, April 17-19, 1994, pp. 65-69.

[Carroll93] Alan Carroll. "ConversationBuilder: A Collaborative Erector Set." Ph.D. Thesis, Department of Computer Science, University of Illinois, 1993.

[ChMa83] J. M. Chang and N. F. Maxemchuk. "A Broadcast Protocol for Broadcast Networks." Proceedings of GLOBCOM (Dec. 1983).

[Chang84] J. M. Chang. "Simplifying Distributed Database Systems Design by Using a Broadcast Network." Proceedings of SIGMOD (June 1984). pp. 223-233.

[ChMa84] J. M. Chang and N. F. Maxemchuk. "Reliable Brosdcast Protocols." ACM Transactions on Computer Systems. 2, 3 (Aug. 1984). pp. 251-273.

[ChZw85] D.R. Cheriton and W. Zwaenepoel. "Distributed Process Groups in the V-Kernel." ACM Transactions on Computer Systems. 3.2 (May 1985). pp. 77-107.

[CrPa88] J. Crowcroft, K. Paliwoda. "A Multicast Transport Protocol". Proceedings of ACM SIGCOMM '88. pp. 247-256.

[Deering89] S. Deering. "Host Extensions for IP Multicasting". STD 5. RFC1112. (August 1989).

[DKM93] D. Dolev, S. Kramer, D. Malki. "Early Delivery Totally Ordered Multicast in Asynchronous Environments." 23rd Annual International Symposium on Fault-Tolerant Computing (FTCS). (Toulouse. France, June, 1993). pp. $544-553$.

[Jacob88] V. Jacobson. "Congestion Avoidance and Control." Proceedings of ACM SIGCOMM '88 Symp (Sept. 1988). pp. 314329.

[JHC94] P. Jain, N. Hutchinson, and S. Chanson. "A Framework or the Non-Monolithic Implementation of Protocols in the $x-$ kernel." Proceedings of USENIX High Speed Networking (August 1994). pp. 13-30. 
[KTHB89] M. F. Kaashoek, A. S. Tanenbaum, S. F. Hummel, H. E. Bal. "An Efficient Reliable Broadcast Protocol." Operating Systems Review. 23, 4 (Oct. 1989), pp. 5-19.

[Lamp78] L. Lamport. "Time, Clocks, and the Ordering of Events in a Distributed System." Communications of the ACM. 21, 7

[LuG190] S. W. Luan and V. D. Gligor. "A Fault Tolerant Protocol For Atomic Broadcast." IEEE Transactions on Parallel and Distributed Systems. 1, 3 (July 1990). pp. 271-285.

[MaBe93] C. Maeda and B. Bershad. "Protocol Service Decomposition for High-Performance Networking." Proceedings of 14th ACM Symposium on Operating Systems Principles, December 1993.

[MaCh84] N. F. Maxemchuk and J. M. Chang. "Analysis of the Messages Transmitted in a Broadcast Protocol." Proceedings of the International Computer Conference (Amsterdam, May 1984). pp. 1263-1267.

[MeBo76] R. M. Metcalf. and D. R. Boggs. "Ethernet: Distributed Packet Switching for Local Computer Networks." Communications of the ACM. 19, 7 (July 1976). pp. 395-404.

[MMA90] P. M. Meillar-Smith. L. E. Moser, V. Agrawala. "Broadcast Protocols for Distributed Systems." IEEE Transactions on Parallel and Distributed Systems. 1, 1 (Jan. 1990). pp. 17-25.

[PEA94] S. Pejhan, A. Eleftheriadis. D. Anastassiou. "Distributed Multicast Address Management in the Global Internet." Submitted to IEEE Journal on Selected Areas in Communication on March 1, 1994.

[PBS89] L. L. Peterson, N. C. Buchholz, R.D. Schlichting. "Preserving and Using Context Information in Interprocess Communication". ACM Transactions on Computer Systems. 7. 3 (Aug. 1989). pp. 217-246.

[Purtilo85] J. Purtilo. "Polylith: An Environment to Support Management of Tool Interfaces." ACM SIGPLAN Symposium on Language Issues in Programming Environments, Seattle. WA, June 25-28, 1985. pp. 12-18.

[RaLi93] K. Ravindran and X. T. Lin. "Structural Complexity and Execution Efficiency of Distributed Application Protocols." Proceedings of ACM SIGCOMM '93. (San Francisco, Sept. 1993). pp. 160-169.

[TNML93] C. Thekkath. T. Nguyen, E. Moy, and E. Lazowska. "Implementing Network Protocols at User Level." IEEE/ACM Transactions on Networking, 1(5), 1993. pp. 554-565.

[Verissmo92] Verissmo. "xAMp: A Multi-primitive Group Communications Service. Proceedings of the 1lth Symposium on Reliable Distributed Computing. 


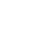

